(Invited paper - Lont. on the Physics of Multiple Charged Ions, Groningen, the Netherlands, Sept. 17-19, 1986 - Proceedings published by NIM)

\title{
DIELECTRONIC RECOMBINATION OF MULTIPLY CHARGED IONS
}

S. DATZ, P. F. DITTNER, C. M. FOU ${ }^{\star}$, F. D. MILLER, and P. L. PEPMILLER Oak Ridge National Laboratory, Oak Ridge, TN 37831-6377 USA

\section{MASTER}

CONF-8609207--1

Abstract

DE87 001689

Using a merged electron-ion merged beam apparatus in conjunction with the ORNL EN Tandem Van de Graaff, we have measured dielectronic recombination in $\Delta n=0$ transitions for a number of Li-like $\left(\mathrm{B}^{2+}, \mathrm{C}^{3+}, \mathrm{N}^{4+}\right.$, and $\left.0^{5+}\right)$, Be-like $\left(\mathrm{C}^{2+}, \mathrm{N}^{3+}\right.$, and $\left.\mathrm{O}^{4+}\right), \mathrm{B}-1 \mathrm{ike}\left(\mathrm{N}^{2+}, \mathrm{O}^{3+}\right.$, and $\left.\mathrm{F}^{4+}\right)$, and $\mathrm{Na-like}$ $\left(\mathrm{P}^{4+}, \mathrm{S}^{5+}\right.$, and $\left.\mathrm{Cl}^{6+}\right)$ ions. The results are compared with theory which includes field enhancement and extension of the more highly charged ions is discussed.

Electron-ion recombination can take place by either of two mechanisms: radiative recombination in which the gain in potential energy is immediately released in the form of a photon and dielectronic recombination (DR) in which a continuum electron excites a previously bound electron and, in so doing, loses just enough energy to be captured into a bound state ( $7 \ell)$. This results in a doubly excited ion of a lower charge state which may either autoionize or emit a photon resulting in a stablized recombination. For an ion $A$ of charge state $q$ in an initial ground state $\alpha$ being excited to a state $\beta$, the $O R$ process can be represented as

$$
A^{q+}(\alpha)+e^{-\left(k, \ell^{\prime}\right) \div\left[A^{(q-1)+}(\beta, n \ell)\right] \star \star+A}(q-1)+(\alpha, n \ell)+h v .
$$

*Permanent address: Dept. of Physics, University of Delaware, Newark, DE. 1971.1. 
The complete signature of the event is an ion of reduced charge and an emitted photon. (It can be distinguished from radiative recombination by the energy dependence of the cross section. A measurement therefore involves the detection of either the photon, the change in charge, or both in coincidence. Measurements of photon production from plasmas have proven quite valuable for $\Delta n=1$ transitions in He-like ions [1] where the second electron is captured into, e.g., the $n=3$ state and the lines are resolvable from simple ionic excitation. For $\Delta n=0$ transitions where most of the DR cross section leads to high $n$ states, this approach is not suitable.

Beam experiments have centered on either charge state change in electron ion collisions as a function of energy or on coincidences of charge state reductions with photons.

The coincidence method using crossed beams has been employed by the JILA group [2] for $\mathrm{Mg}^{+}$and by Williams [3] for $\mathrm{Ca}^{+}$. Moreover, the JILA group has done differential field ionization studies on the recombined $\mathrm{Mg}^{\circ}$ that have revealed features of the $\mathrm{n}$ distribution obtained in the process $[4]$.

A second approach using merged electron-ion-beams to attain low center-of-mass collision energies has been used by two groups. Mitchell and co-workers [5] at Western Ontario University used this method on $\mathrm{C}^{+}$. The electron beam used there was emission limited with a small energy spread; but was accordingly quite tenuous, resulting in rather weak signal levels.

The only results obtained thus far for multiply charged ions have been those of the 0ak Ridge group $[6,7]$. The approach of the Oak Ridge group is 
to use high velocity multiply charged ion beams merged with a high-density compressed space charge limited electron beam as shown schematically in fig. 1. The advantages are that (1) multicharged ions are easily produced at high velocities obtained from e.g., tandem Van de Graaff accelerators, (2) charge transfer cross sections on background gas for multicharged ions are reduced by $\sim 4$ orders of magnitude at MeV/nucleon energies compared to $\mathrm{keV} /$ nucleon energies, and (3) higher ion velocities require higher energy electron beams and hence higher space charge limited currents and electron target densities. This approach leads to ease of measurement and results have been obtained for all the ion species listed in Table 1.

In these experiments, the ion beam from the ORNL EN Tandem Accelerator enters the interaction region through an axial, $0.64 \mathrm{~mm}$ diameter hole in the cathode of the electron gun. After exiting the interaction region, the ion beam is subjected to charge state analysis. The initial charge state of the ion beam, $q+$ is deflected into a Faraday cup. The cup is connected to a current integrator and the output pulses are counted by a scaler. Ions that have picked up en electron (charge $=(q-1)+$ ) are deflected onto a solid state position sensitive detector (PSD). The ions having charge $(q-1)+$ arise from electron pickup of the $q+$ ions from the residual gas, slit edge scattering, and the sought after effect, DR. (Further details of the experiment are given in ref. [7].

The source of the electron beam is a double gridded Pierce-type highintensity electron gun which is designed to produce a convergent, laminar electron beam. The gun is operated in the space charge limited mode. The electron beam enters a coaxial solenoidal magnetic field which is adjusted to establish Brillouin flow (e.g., $0.18 \mathrm{~T}$ for $1 \mathrm{keV}$ electrons) 
in which the beam radius $(1.6 \mathrm{~mm})$ stays constant and the beam rotates as a solid of revolution about its axis with the Larmor frequency, $\omega_{L}$. Under Brillouin flow, the longitudinal velocity of an electron is independent of radius, the radial velocity is zero, and the azimuthal velocity is equal to $\omega_{L}$ times the radial position of a particular electron. Surrounding the electron beam is a coaxial cylinder $84 \mathrm{~cm}$ long having an inside diameter of $7.9 \mathrm{~mm}$, which is normally electrically grounded. Following the interaction region, defined by the length of the coaxial cylinder and the solenoidal field, the electrons expand (due to space charge repulsion) and strike the chamber walls.

The experimental procedure consisted of optimizing the electron beam, at a particular cathode voltage and counting the $\mathrm{A}(\mathrm{q}-\mathrm{l})+$ and $\mathrm{Aq}^{+}$beams while stepping through the relative energies of interest by changing the energy of the ion beam.

In order to measure signal and background, we modulate the voltage applied to the guide tube surrounding the merged beam region. For half the time the tube is grounded and for the other half the tube is raised in voltage such that the electron beam energy is shifted to be off resonance. The result of such an experiment is shown for $\mathrm{s}^{5+}$ in fig. 2. Subtracting point-by-point removes the jumps in the data which occur due to differences in slit edge scattering and give the result shown in fig. 3 . After a small correction for the background modulation offset, we are left with the signal ratio $R_{S}$. $R_{S}$ is related to the DR cross section $\sigma$, by

$$
\left.R_{S}=\iiint v_{r} \sigma\left(v_{r}\right) \rho_{e}\left(v_{r}\right) \rho_{i} d v_{r} d V\right) / \int \rho_{i} v_{j} d A
$$

where $\rho_{e}$ is the electron density, $\rho_{j}$ is the ion density, $v_{r}$ is the relative 
velocity, $v_{i}$ is the velocity of the ions, and $V$ and $A$ are the volume and cross sectional area of the interaction region, a cylinder whose radius is that of the ion beam $r_{j}$, and having the length of the electron beam, $L$. Since $\rho_{i}$ and $v_{i}$ are constant within $v_{\text {, the }}$ ion current $I_{i}=\int \rho_{i} v_{i} d A=$ $\rho_{j} v_{j} A$. Approximating $\rho_{e}$ by an average electron density $\rho_{e}$, times a distribution in relative velocities $f\left(v_{r}\right)$, both being independent of position within $v$, we can write, $R_{S}=\left(\rho_{\mathrm{e}} \mathrm{L}\left\langle v_{r} \sigma\right\rangle\right) / v_{j}$, where the triangular brackets denote the average over $f\left(v_{r}\right)$. Thus, from the measured quantities we can calculate $\left\langle v_{r} \sigma\right\rangle$ at every ion energy or relative energy, i.e., $\left\langle v_{r} \sigma\right\rangle=$ $R_{S} v_{j} / \rho_{e} L$. The $\left\langle v_{r} \sigma\right\rangle$ versus $E_{r}$ data for $S^{5+}$ are shown in fig. 4.

For $S^{5+}$, we expect a very sharp peak (almost a $\delta$ function on our scale) at the Rydberg limit of $n \ell$. The observed width is due to electron beam energy resolution. Using a two-parameter fit, one describing the longitudinal and the second the transverse distribution, we find we are able to fit the rates. The same two parameters fit for all the ions used which have single $\delta$ runction in their DR cross sections $\left(B^{2+}, C^{3+}, N^{4+}\right.$, $0^{5+}, \mathrm{P}^{4+}, \mathrm{S}^{5+}$, and $\mathrm{Cl}^{6+}$ ) thus giving us some confidence that we have now characterized our electron beam.

The theoretically predicted probability of recombination observed in these experiments depends on the electric fields present in two regions: first, the electron-ion interaction region and second, the charge analysis region.

The electric fields in the charge analysis region can field ionize Rydberg states formed in the electron-ion interaction. The maximum $n$ 
state that can survive these fields is given by

$$
n_{\max }=\left(6.31 \times 10^{8} q^{3} / E\right)^{1 / 4}
$$

where $q$ is the core charge and $E$ is the field in volts per $\mathrm{cm}$. In the present measurements, we use an analyzing field of $\sim 4 \mathrm{kV} / \mathrm{cm}$.

Less well understood is the effect of small fields in the interaction region which mix $\ell$ states for a given $n$ and can greately increase the DR cross section. This effect can be easily understood from the following considerations. The dielectronic recombination cross section oDR is given by

$$
\sigma_{D R} \propto \sigma^{\star \star} \frac{A_{r}}{A_{a}+A_{r}}
$$

where $\sigma^{\star \star}$ is the cross section for formation of the doubly excited state of eq. (1), $A_{r}$ is the radiative rate leading to stabilization of this state, and $A_{a}$ is the autoionizing rate. But since $\sigma^{\star \star} \propto A_{a}$,

$$
\sigma_{D R} \propto A_{r} A_{a} /\left(A_{r}+A_{a}\right)
$$

and hence large $\sigma_{D R}$ is expected for $A_{a} \gg A_{r}$, where $\sigma_{D R} \propto A_{r}$.

With this in mind consider the $\ell$ dependence of $A_{a}$ for a given $n$ : An example, $\mathrm{Mg}^{+} \mathrm{n}=20$, is shown [8] in fig. 5. The radiative rate $A_{r}$ for the $\beta \rightarrow \alpha$ core relaxation is essentially independent of $\ell$, but $A_{a}$ falls rapidly with $\ell$. If we make the assumption that all states with $A_{a}>A_{r}$ contritute to ${ }^{\circ} D R$ and all siates with $A_{a}<A_{r}$ do not, we find a total of 72 states which contribute. If we place the ion into a field strong enough to mix all the $\&$ states, we must now use the Stark representation [8] shown in 
fig. 6. Using the same criteria as for fig. 5, we find that 304 states should read to $D R$; a gain in $\sigma_{D R}$ of a factor of $\sim 4.2$.

The solid curves in fig. 4 represent the calculated cross sections with an appropriate high $n$ cutoff (eq. (3)) due to the aralyzing field and a fold of our known energy distribution. The upper curve is for a totally Stark-mixed (high field) case and the lower represents an unmixed (zero field) case. The data fall in between and can be fit with a field of $\sim 25 \mathrm{~V} / \mathrm{cm}$. This same fit with theory, assuming a $25 \mathrm{~V} / \mathrm{cm}$ field, holds for the other Na-like ions $\mathrm{P}^{4+}$ and $\mathrm{Cl}^{6+}$ which we have measured [7]. The field in our apparatus arises from the voltage drop across our space-charge limited electron beam. The value of $25 \mathrm{~V} / \mathrm{cm}$ would appear to be a bit high but it is not impossible for our conditions.

The data for Li-like ions is typified by the $B^{2+}$ data shown in fig. 7 . Here, the theory would require fields of several hundred volts per centimeter in order to fit the data. This is clearly out of the range of possibility. Again, as in the case of Na-like ions, the data for Li-like ions which we have measured $\left(\mathrm{B}^{2+}, \mathrm{C}^{3+}, \mathrm{N}^{4+}\right.$, and $\left.\mathrm{O}^{5+}\right)$ are consistent with each other but for the theory to fit in this case all would require total Stark saturation [9].

In the case of Be-like ions $\left(\mathrm{C}^{2+}, \mathrm{N}^{3+}, \mathrm{O}^{4+}\right)$, a new element is introduced; the presence of metastables in the beam. The ground state ions may recombine via:

$$
1 s^{2} 2 s^{2}{ }^{1} s+e \neq\left[1 s^{2} 2 p^{2}{ }^{1} p n l\right]^{\star *}+\left[1 s^{2} 2 s^{2}{ }^{1} s n l\right]^{\star}
$$

For the metastable, the possibilities are: 


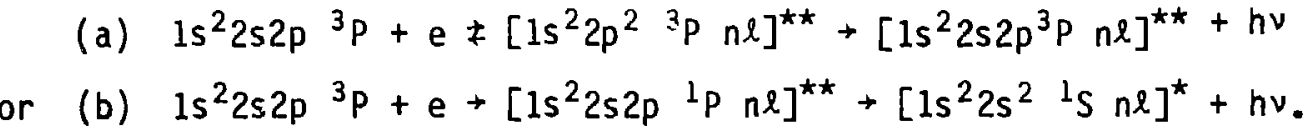

Path (a) is not expected to be significant because the radiatively relaxed ${ }^{3} \mathrm{P} n \ell$ state is still capable of autoionization. Path (b), involving the triplet-singlet transition, shows surprisingly great strength in the coupled-channel calculations of Griffin and co-workers [11]. The tripletsinglet cross section of the metastable should be lower than that for the ground state excitation but the beam composition is $~ 70 \%$ metastable [10]. The data for $\mathrm{N}^{3+}$ is shown in fig. 8 together with calculations [11] for zero field and complete Stark mixing of states. No calculations yet exist for estimating the "required field" for fit.

In fig. 8 , we show the DR rate for Boron-like $0^{3+}$. Here, three intermediate excited states are possible $0^{3+}\left(1 s^{2} 2 s^{2} 2 p^{2} P\right)+e \neq$ $\left[0^{2+}\left(1 s^{2} 2 s 2 p n l\right)^{2} D,{ }^{2} S \text { or }{ }^{2} \mathrm{P}\right]^{\star \star}$. In addition to the $D R$ path to $\left[0^{2+} 1 s^{2} 2 s^{2} 2 p{ }^{2} P n l\right]^{*}+h v$, the ${ }^{2} S$ and ${ }^{2} p$ states have alternative autoionizing paths to $\left[0^{3+}\left(1 s^{2} 2 s 2 p^{2} 2 D\right)\right]^{+}+e$ and the $2 p$ state has yet another autoionizing path to $\left[0^{3+}\left(1 s^{2} 2 s 2 p{ }^{2} S\right)\right]^{*}+e$. These alternative autoionizing paths steal strength from the DR channels for the ${ }^{2} S$ and ${ }^{2} p$ doubly excited intermediates. In fig. 9, we show a fit to the data using a knowledge of where the resonances for the three states should appear and folding the known energy resolution function to each. The fit is obtained simply by varying the amounts of the three components. The B-1ike ion beams used consisted of $\sim 50 \%$ metastable $1 s^{2} 2 s 2 p^{2}{ }^{4} p$ ions [10] mixed with the stable ground state. It is expected, however, that the 
metastables do not contribute appreciably to the signal. The detailed theory for the B-like ions has not yet been deveioped.

The limitations of the setup we presently use are: resolution of only $\sim 5 \mathrm{eV}$, background levels due to gas and slit edge scattering, and the limitations of ion species (charge state) ion species, and velocity available from our EN tandem Van de Graaff. Only in the case of B-like ions do individual resonances or, more accurately, sets of resonances become resolvable. Future directions include higher resolution, higher charge state and $\Delta n \neq 0 \alpha+\beta$ transitions. Some interesting predictions [11] can be seen in fig. 10 which shows the anticipated spectrum from Li-like Fe $\mathrm{F}^{3+}$ via $2 s+2 p(\Delta n=0)$ excitation and in fig. 11 for $2 s+3 \ell(\Delta n=1)$ excitation. In both cases, an instrumental (Gausisian) resolution of $3 \mathrm{eV}$ has been folded in and the effects of Stark mixing of $\ell$ states are shown. For the higher charge states, the major contributions to the DR cross sections no longer come from $2 p \rightarrow 2 s$ relaxation with capture to high $\mathrm{n}$ states. Instead, the radiative rates for relaxation of the lower $n$ states are now sufficiently high to be the dominant path to recombination. The lines in the spectrum now become resolvable even with 3-5 eV resolution and the field effect is small. For the $\Delta n=1$ transition shown in fig. 11, the same is true, but the cross section is reduced by a factor of 40 compared to the $\Delta n=0$ process. Measurements of these latter cross sections may have to await beam luminosities that will become available with the advent of heavy ion storage rings.

This research was sponsored by the U.S. Department of Energy, Division of Chemical Sciences, Office of Basic Energy Sciences under Contract No. DE-AC05-840R21400 with Martin Marietta Energy Systems, Inc. 


\section{References}

[1] M. Bitter, K. W. Hill, N. R. Sauthoff, P. C. Efthimion, E. Meservey, W. Roney, S. von Goeler, R. Horton, M. Goldman, and W. Stodiek, Phys. Rev. Lett. $\underline{43}$ (1979) 129.

[2] D. S. Belíc, G. H. Dunn, T. J. Morgan, D. W. Mueller, and C. Timmer, Phys. Rev. Lett. 50 (1983) 339.

[3] J. F. Williams, Phys. Rev. A29 (1984) 2936.

[4] A. Müller, D. S. Belíć, B. D. DePaola, N. Djuríc, G. H. Dunn, D. W. Mueller, and C. Timmer, Phys. Rev. Lett. $\underline{56}$ (1986) 127.

[5] J.B.A. Mitche11, C. T. Ng, J. L. Forand, D. P. Levac, R. E. Mitchell, A. Sen, D. B. Miko, and J. W. McGowan, Phys. Rev. Lett. $\underline{50}$ (1983) 335.

[6] P. F. Dittner, S. Datz, P. D. Miller, C. D. Moak, P. H. Stelson, C. Bottcher, W. B. Dress, G. D. Alton, N. Nesković, and C. M. Fou, Phys. Rev. Lett. 51 (1983) 31.

[7] P. F. Dittner, S. Datz, P. D. Miller, P. L. Pepmiller, and C. M. Fou, Phys. Rev. A33 (1986) 124. Also in "Atomic Excitation and Recombination in External Fieids," H. M. Nayfeh and C. W. Clark, eds., Gordon and Breach, New York, 1985.

[8] D. C. Griffin, M. S. Pindzola, and C. Bottcher, Oak Ridge National Laboratory Report No. ORNL/TM-9478, 1985.

[9] P. F. Dittner, S. Datz, P. D. Miller, P. L. Pepmiller, and C. M. Fou, to be published.

[10] S. Datz, P. F. Dittner, H. F. Krause, P. D. Miller, P. L. Pepmiller, Y. Yamazaki, and N. Stolterfoht, Bull. Am. Phys. Soc. $\underline{31}$ (1986) 974.

[11] D. C. Griffin, M. S. Pindzola, and C. Bottcher, private communication. 


\section{Figure Captions}

Fig. 1. Schematic Diagram of Merged Beam Apparatus.

Fig. 2. Ratio $\left(\mathrm{S}^{4+} / \mathrm{S}^{5+}\right)$ as a function of $\mathrm{S}^{5+}$ ion energy (top) and relative energy (bottom) for a $617 \mathrm{eV}$ merged electron and ion beam. The lower curve shows the effect of the offset in electron beam energy at the same ion energy.

Fig. 3. Difference signal of $\mathrm{S}^{4+} / \mathrm{S}^{5+}$ ratio from fig. 2 .

Fig. 4. DR rate $v_{r} \bar{\sigma}$ vs $E_{r}$ for $s^{5+}$. The upper curve is calculated from ref. [8] with complete Stark mixing of $\ell$ states and lower curve with zero field.

Fig. 5. $A_{a}(n, l)$ and $A r$ vs. $\ell$ in spherical coordinates for $n=20$ in $\mathrm{Na}-$ like $\mathrm{Mg}^{+}$.

Fig. 6. $A_{a}(n, k, m)$ and $A r$ vs. $k$ in parabolic coordinates for $n=20$ in $\mathrm{Na-like} \mathrm{Mg.} \mathrm{The} \mathrm{number} \mathrm{to} \mathrm{the} \mathrm{right} \mathrm{of} \mathrm{each} \mathrm{curve} \mathrm{drawn} \mathrm{through}$ the points equals $|\mathrm{m}|$.

Fig. 7. DR rate $v_{r} \bar{\sigma}$ vs. $E_{r}$ for $B^{2+}(L i-l i k e)$. Points are the experimental data. The curves represent the theory for differing electric fields in the recombination region $(\ldots 0 \mathrm{~V} / \mathrm{cm}, \ldots 5 \mathrm{~V} / \mathrm{cm}$, _.___ $25 \mathrm{y} / \mathrm{cm}, \ldots \ldots 125 \mathrm{~V} / \mathrm{cm}$, and _... $625 \mathrm{~V} / \mathrm{cm})$.

Fig. 8. DR rate $v_{r} \bar{\sigma}$ vs. $E_{r}$ for $N^{3+}$ (Be-like). Points are experimental data, the curve represents theory with complete Stark mixing of $\ell$ states and the lower curve zero field.

Fig. 9. DR rate $v_{r} \bar{\sigma}$ vs. $E_{r}$ for $0^{3+}(B-1 i k e)$. Fitted curve obtained from addition of ${ }^{2} S,{ }^{2} p$ and ${ }^{2} D$ contributions. 
$-12-$

Figure Captions (contd)

Fig. 10. Calculated DR cross section for $\mathrm{Fe}^{23+}(\mathrm{LI}-\mathrm{like})$ in the $2 \mathrm{~s}+2 \mathrm{p}$ $(\Delta n=0)$ region. Results shown are for an experimental resolutron of $3 \mathrm{eV}$ (Gaussian). Field enhancement with complete $\ell$ mixing is shown by the dashed line (ref. [11]).

Fig. 11. Calculated DR cross section for $\mathrm{Fe}^{23+}(\mathrm{Li}-1 \mathrm{like})$ in the $2 \mathrm{~s}+3 l$ $(\Delta n=1)$ region. Results shown are for an experimental resolutron of $3 \mathrm{eV}$ (Gaussian). Field enhancement with complete \& mixing is shown by the dashed line (ref. [11]). 
Table I

Dielectronic recombination of multicharged ions studied by the ORNL group

$$
\Delta \mathbf{n}=0 \text { transitions in: }
$$

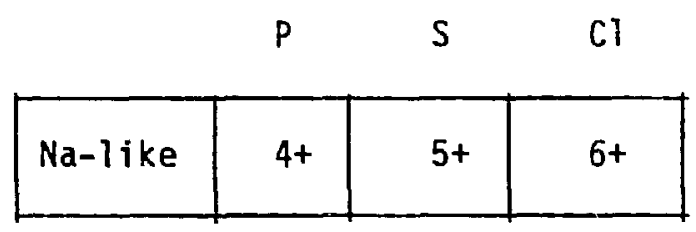

\begin{tabular}{|l|l|l|l|l|l|}
\multicolumn{2}{c}{ B } & C & N & 0 & F \\
\hline Li-like & $2+$ & $3+$ & $4+$ & $5+$ & \\
\hline Be-like & & $2+$ & $3+$ & $4+$ & \\
\hline B-like & & & $2+$ & $3+$ & $4+$ \\
\hline
\end{tabular}




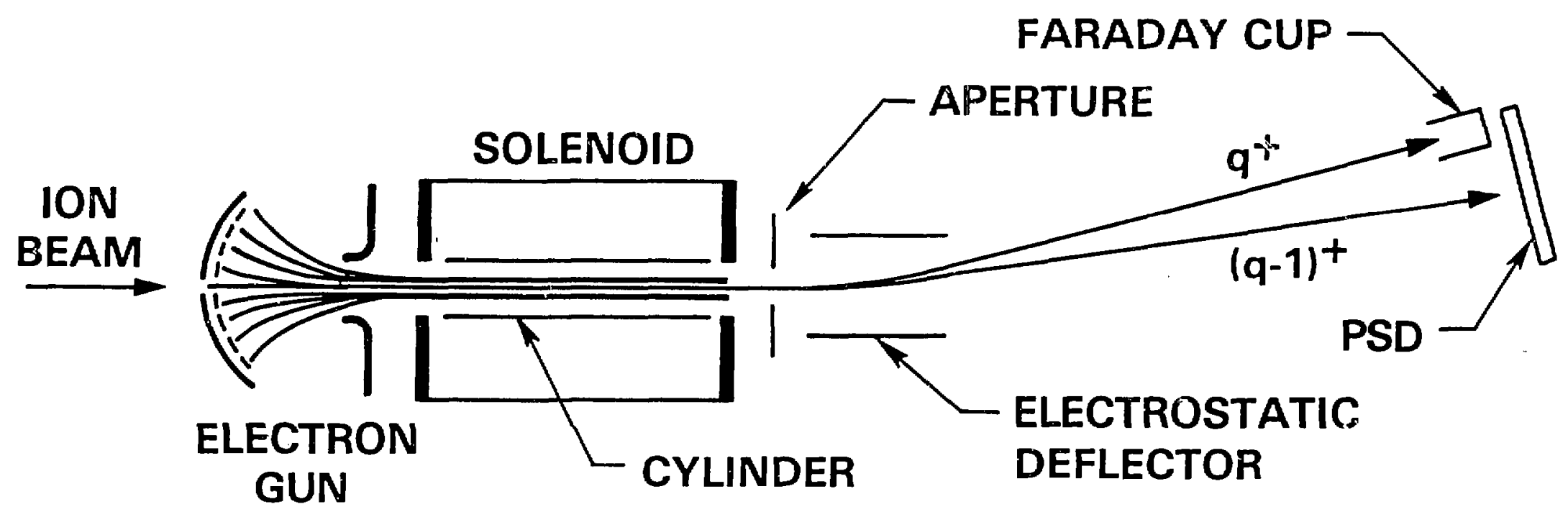

Fig. 1 


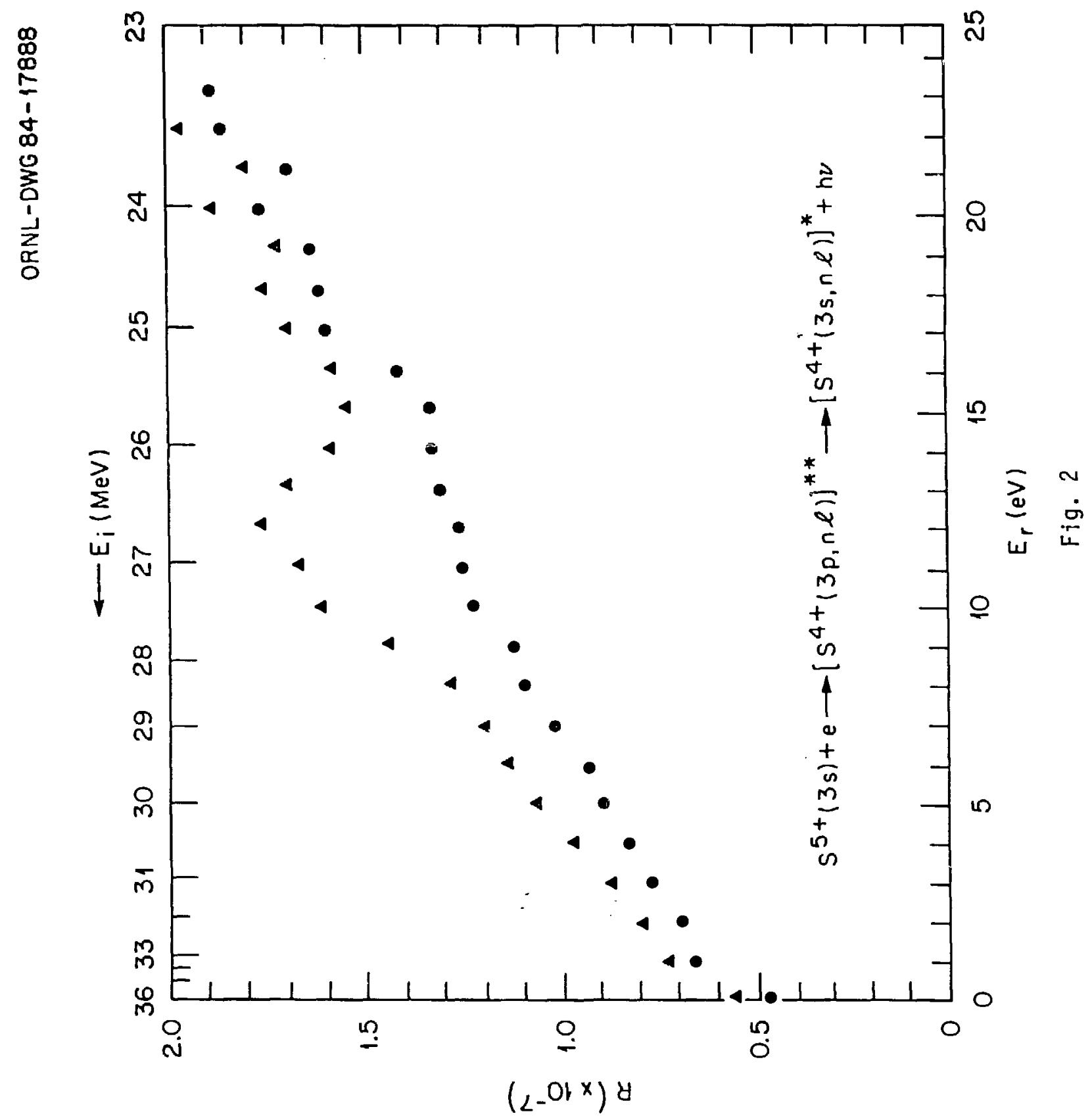




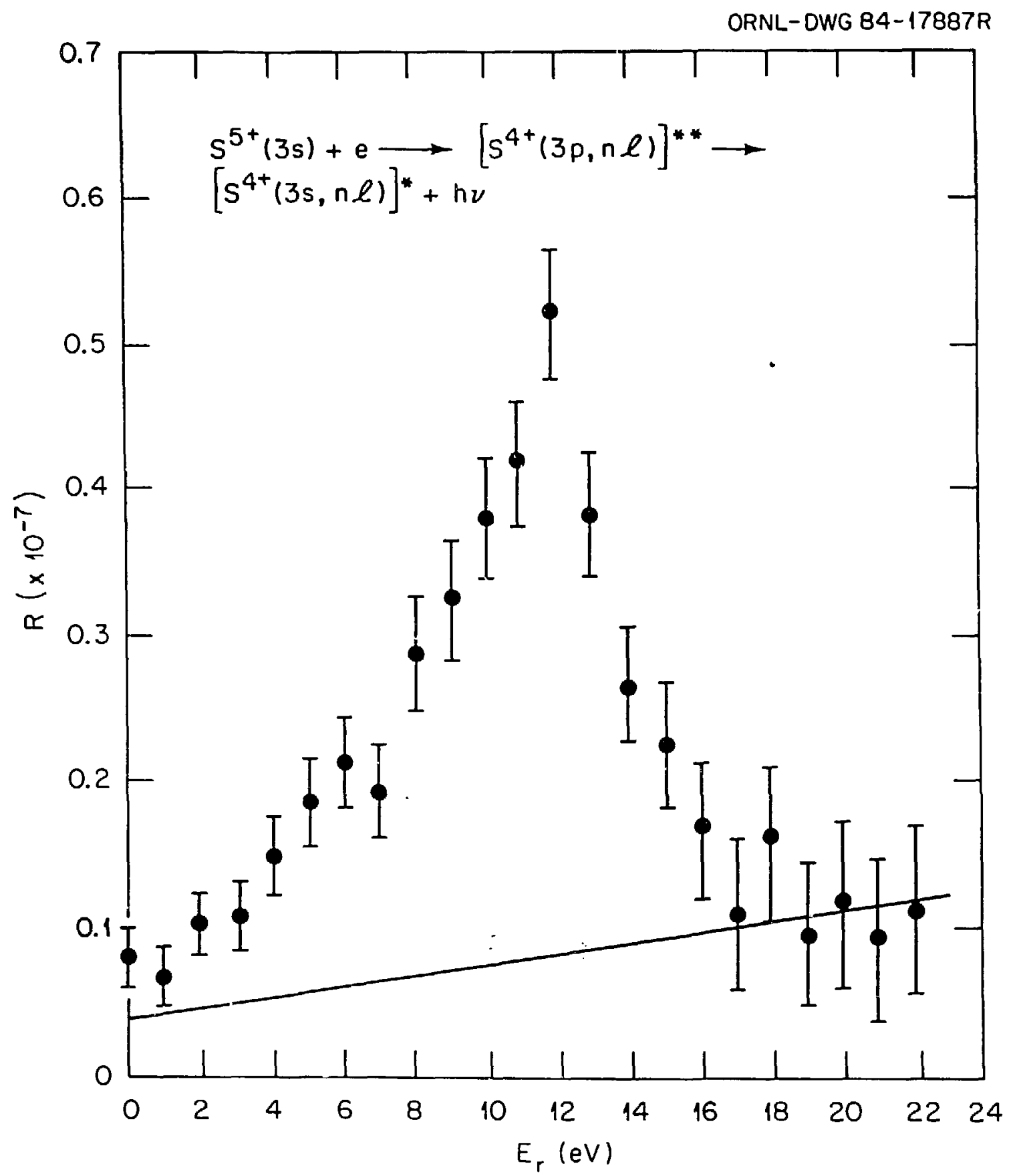

Fig. 3 


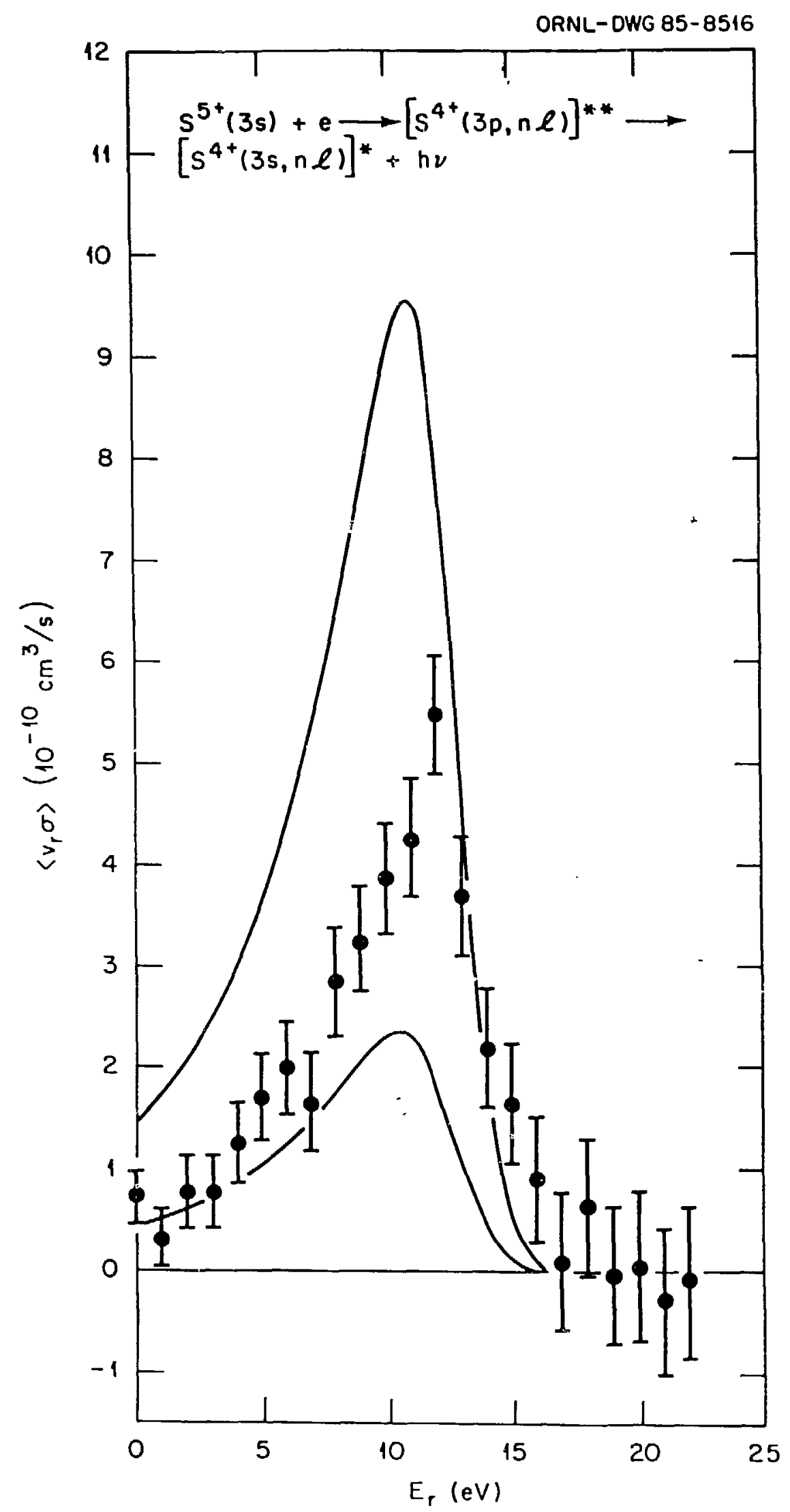

Fig. 4 


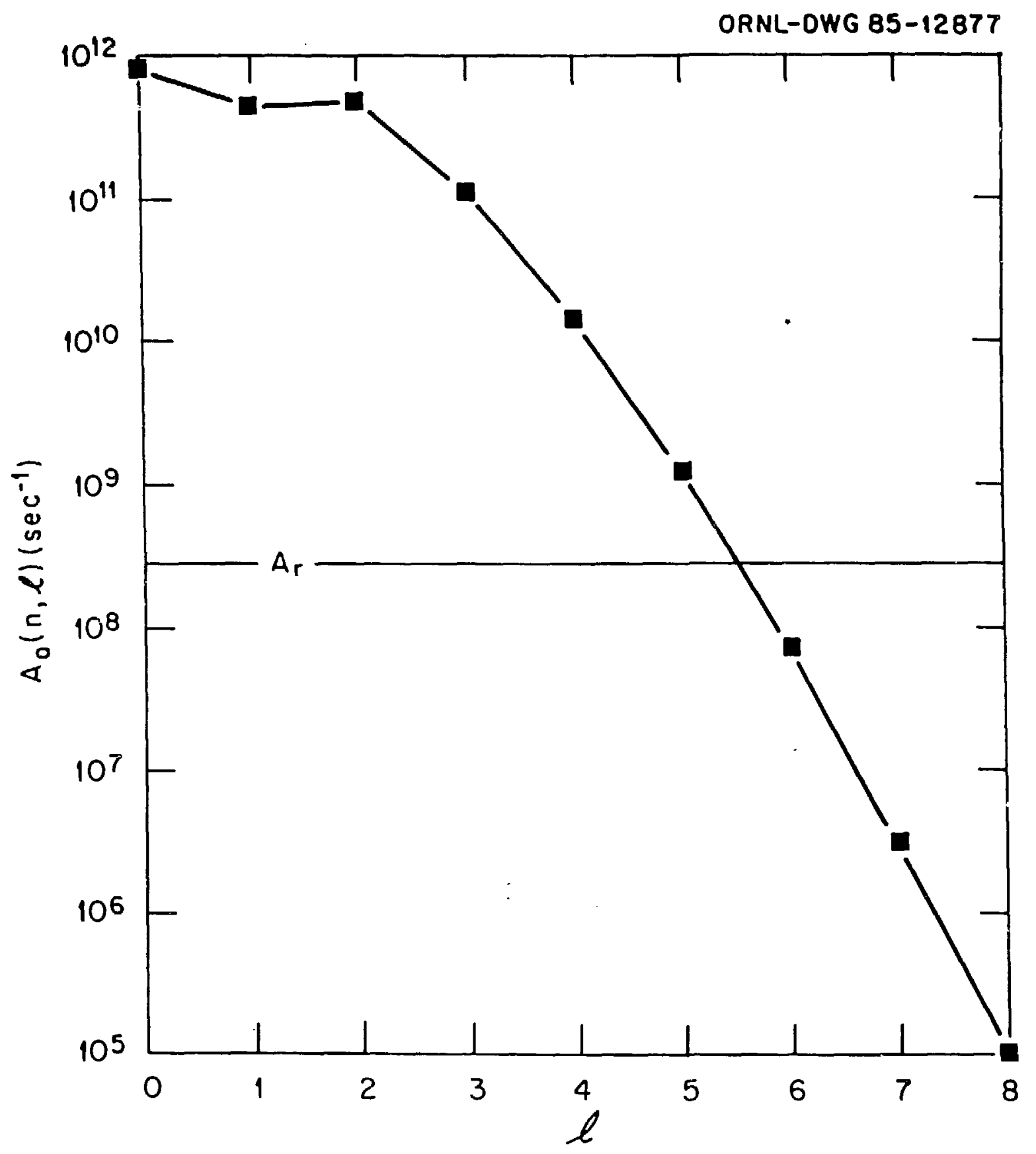

Fig. 5 


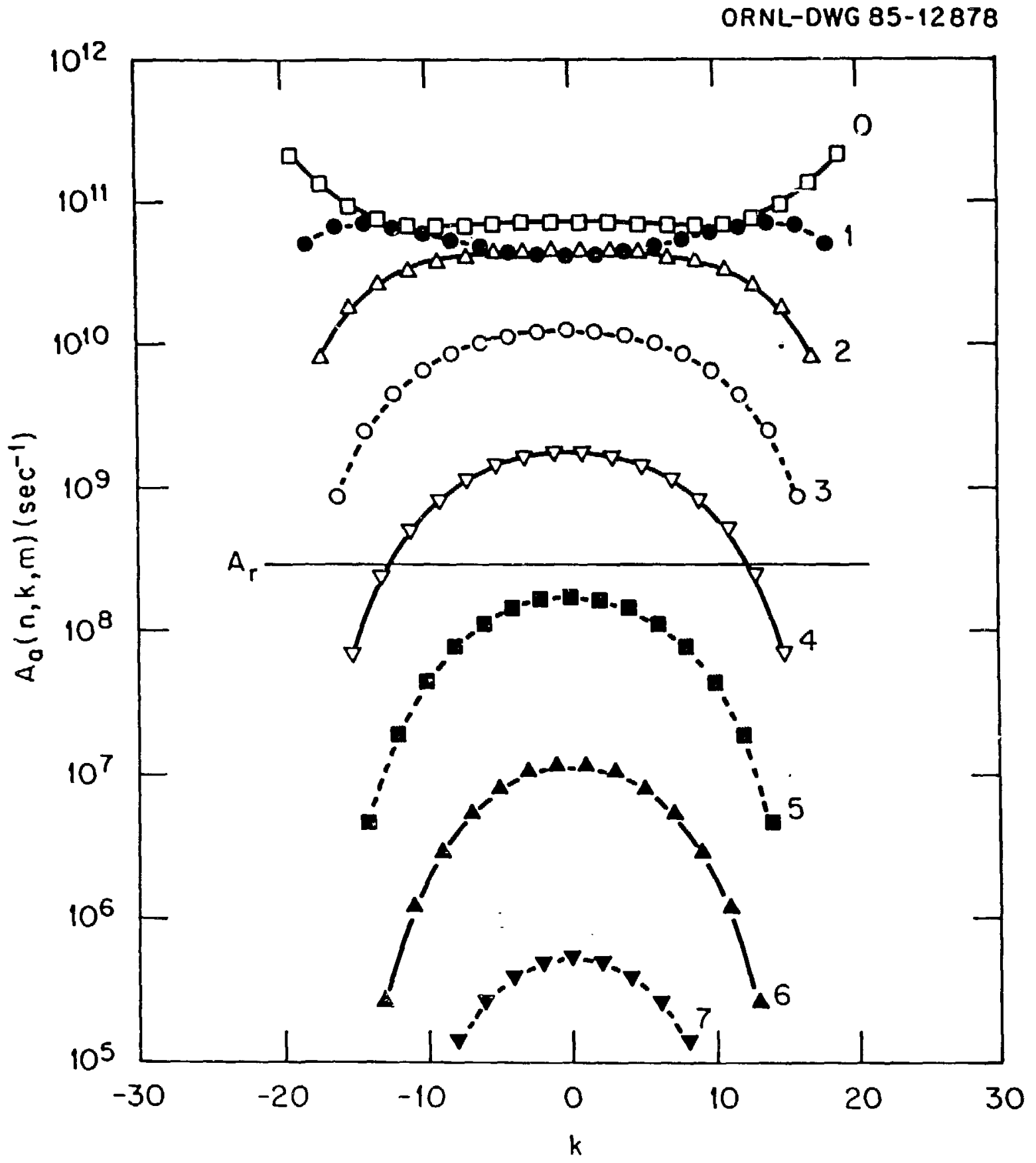

Fig. 6 


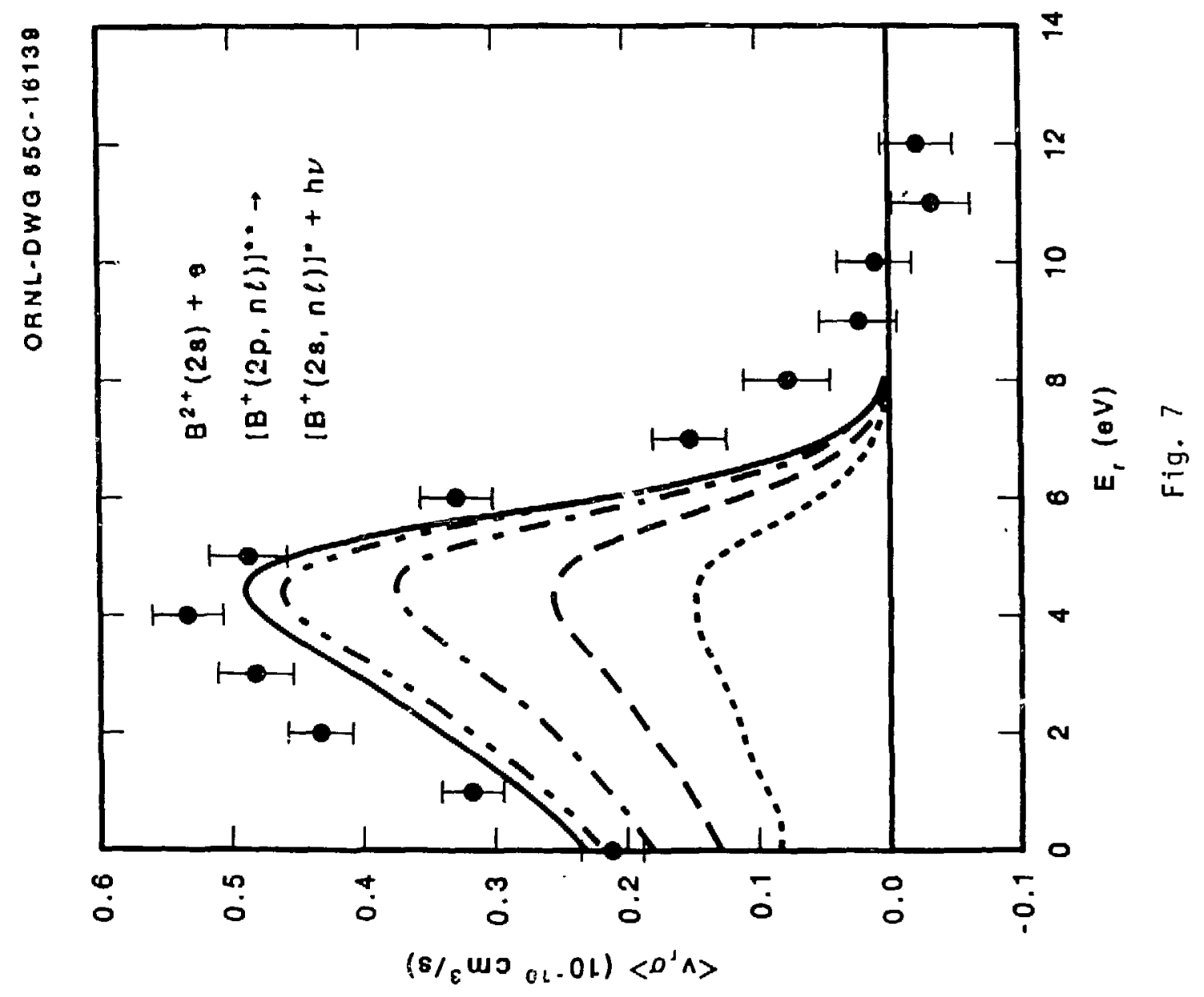




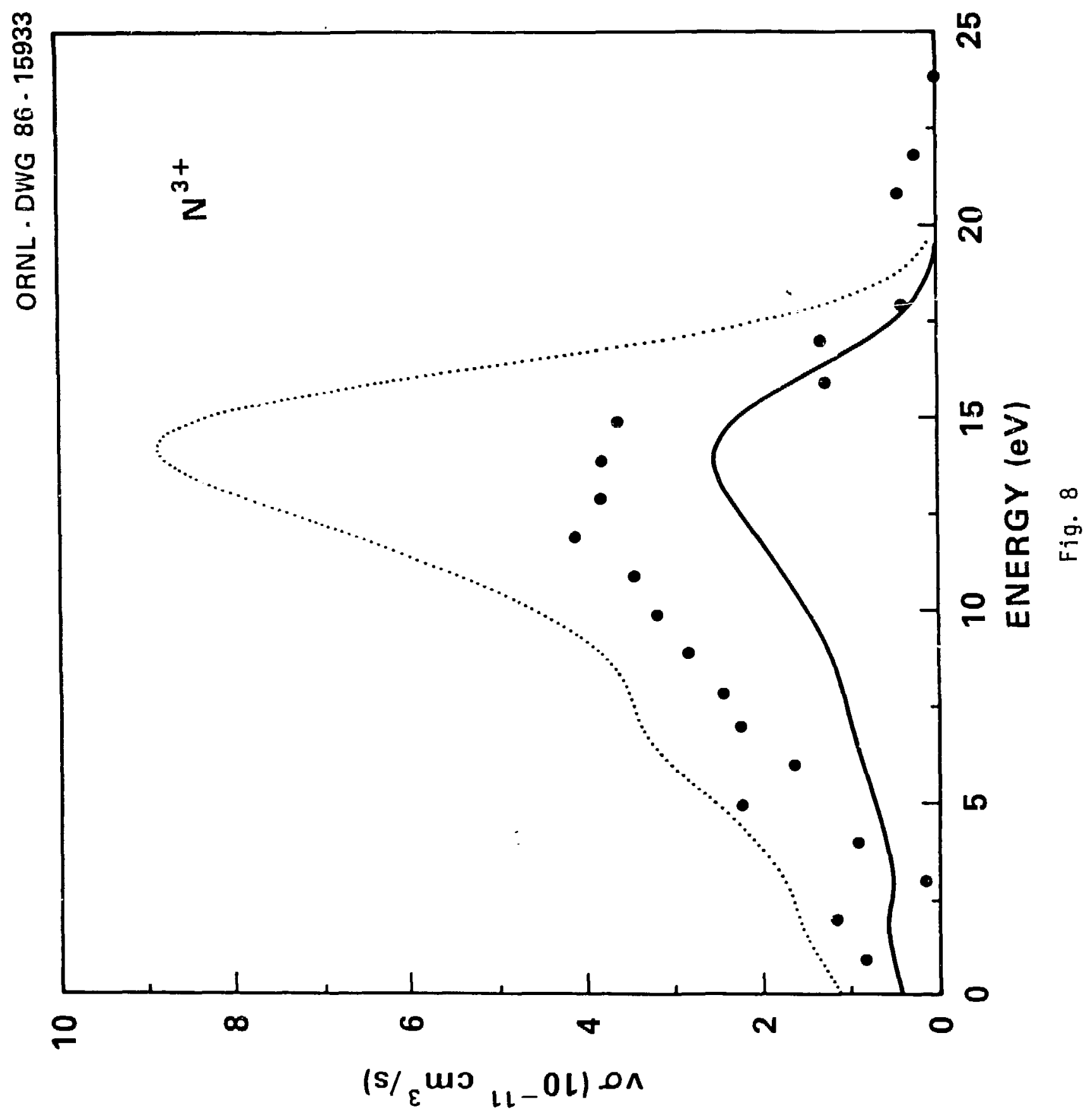




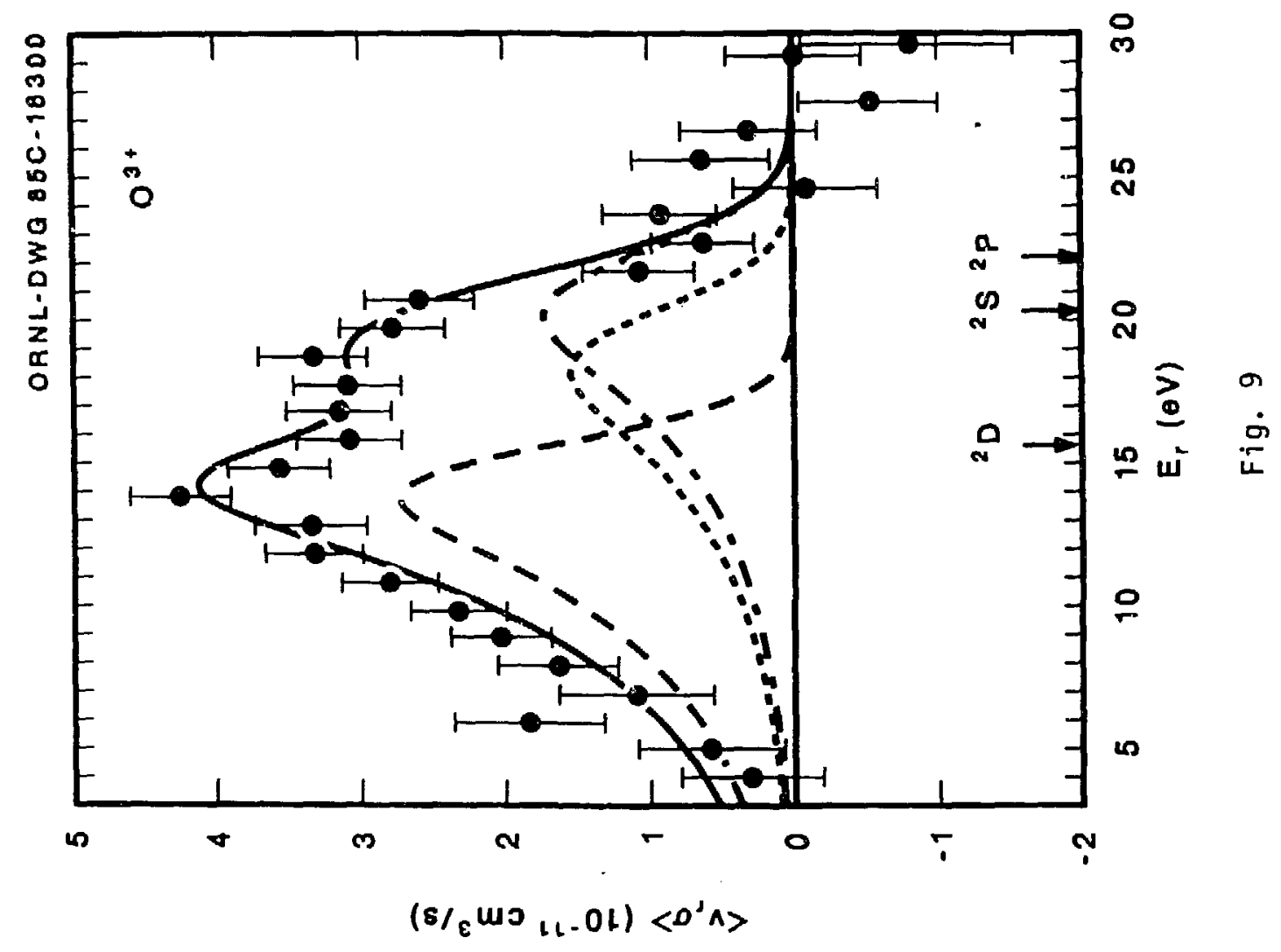




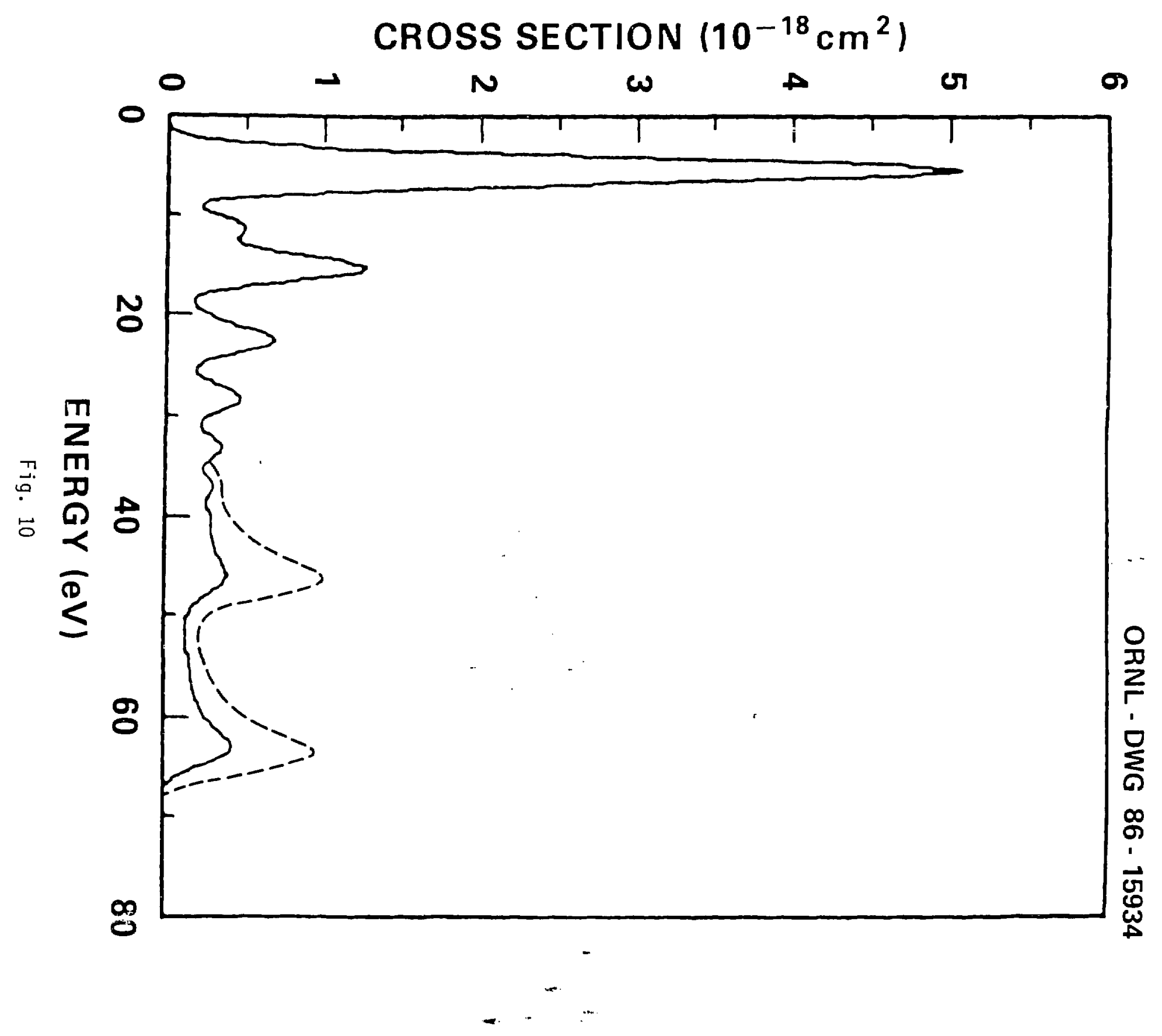




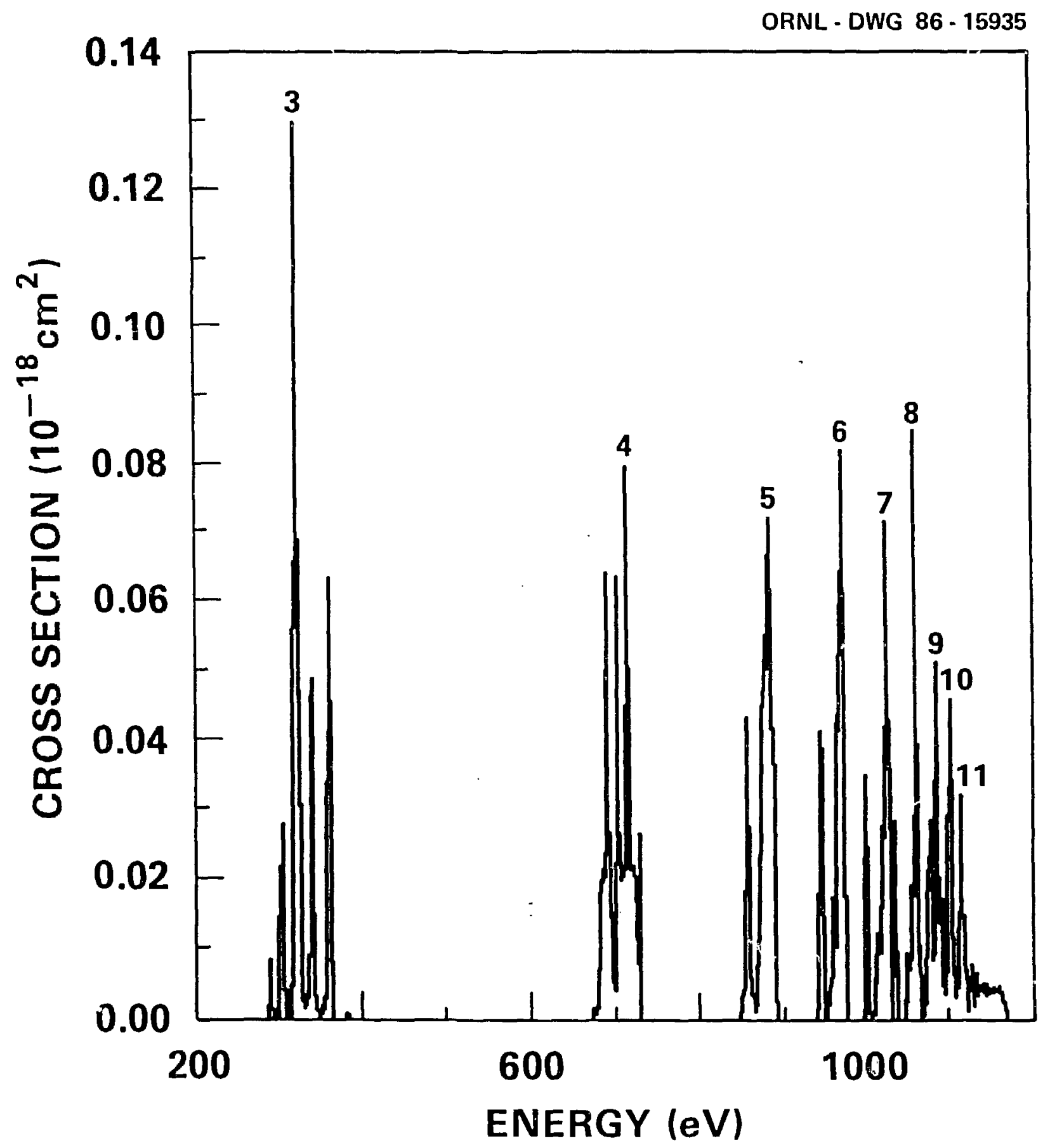

Fig. 11 


\section{DISCLAIMER}

This report was prepared as an account of work sponsored by an agency of the United States Government. Neither the United States Government nor any agency thereof, nor any of their employees, makes any warranty, express or implied, or assumes any legal liability or responsibility for the accuracy, completeness, or usefulness of any information, apparatus, product, or process disclosed, or represents that its use would not infringe privately owned rights. Reference herein to any specific commercial product, process, or service by trade name, trademark, manufacturer, or otherwise does not necessarily constitute or imply its endorsement, recommendation, or favoring by the United States Government or any agency thereof. The views and opinions of authors expressed herein do not necessarily state or reflect those of the United States Government or any agency thereof. 